\title{
Survivin is expressed in early hepatocellular carcinoma and surrounding hepatitis tissue
}

\author{
SATOSHI MAMORI ${ }^{1}$, MASATO MATSUSHIMA ${ }^{2}$, TOMOKAZU MATSUURA $^{3}$ and HISAO TAJIRI ${ }^{1}$
}

\begin{abstract}
Divisions of ${ }^{1}$ Gastroenterology and Hepatology, Department of Internal Medicine, and ${ }^{2}$ Clinical Research and Development,
${ }^{3}$ Department of Laboratory Medicine, The Jikei University School of Medicine, Tokyo 201-8601, Japan
\end{abstract}

Received November 19, 2008; Accepted May 7, 2009

DOI: 10.3892/mmr_00000191

\begin{abstract}
It has been suggested that survivin, a member of the inhibitor of apoptosis protein (IAP) family, is an attractive novel target for anticancer therapy. The present study investigated the expression of survivin during the early stages of hepatocellular carcinoma (HCC) and associated hepatitis, and attempted to elucidate how this expression is correlated with clinicopathological factors. Twenty-two patients (15 men and 7 women) underwent a liver tissue biopsy for the diagnosis of HCC. In the HCC and surrounding hepatitis-infected tissues, the average survivin expression rate was 62.36 and $31.41 \%$ (median), respectively. The serum level of ALT was correlated with the survivin expression rate in the $\mathrm{HCC}(\mathrm{r}=-0.60, \mathrm{P}<0.01)$ and hepatitis specimens $(\mathrm{r}=-0.43, \mathrm{P}<0.05)$. The data suggest that certain clinicopathological factors may in future serve as useful indicators for the selection of patients responsive to the inhibition of survivin.
\end{abstract}

\section{Introduction}

Hepatocellular carcinoma (HCC) is the fifth most frequent cancer and the third leading cause of cancer-related death in the world, with an estimated annual prevalence of $>5,000,000$ cases worldwide (1). Most patients with HCC have no indications for any specific therapy strategies, such as surgery, percutaneous ethanol injection therapy and radiofrequency ablation, due to multiple recurrences with small nodules (2). As a result, patients must undergo interventional radiology (IVR), such as transcatheter arterial chemoembolization (TACE). A more effective IVR drug is therefore required.

Survivin is a recently identified member of the inhibitor of apoptosis protein (IAP) family (3) that is strongly associ-

Correspondence to: DrSatoshi Mamori,Division of Gastroenterology and Hepatology, Department of Internal Medicine, Daisan Hospital, The Jikei University School of Medicine, 4-11-1 Izumihoncho, Komaeshi, Tokyo 201-8601, Japan

E-mail:mamori@jikei.ac.jp

Key words: survivin, hepatocellular carcinoma, hepatitis, alanine aminotransferase ated with apoptosis, cell proliferation and cell cycle control (4-7). By inhibiting apoptosis and promoting mitosis, survivin facilitates cancer cell survival and growth (6,8-11). Survivin is selectively expressed in the most common human neoplasms, and appears to be involved in tumor cell resistance to some anticancer agents and ionizing radiation (12).

A previous report suggests that survivin inhibition in early HCC is potentially useful as an effective interventional radiological treatment modality (13). This study investigated the expression of survivin in early-stage HCC tissue and in its surrounding hepatitis-infected tissue, and examined the correlation of this expression with clinicopathological factors.

\section{Materials and methods}

Patients. Between January 2003 and December 2005, 22 patients (15 men and 7 women; median age 68 years, range 56-81 years) underwent a concurrent tumor and non-tumor liver tissue biopsy for the diagnosis of HCC at the Jikei University Daisan Hospital, Tokyo, Japan (Table I). Three peritumoral liver tissue specimins of metastatic liver cancer (pancreatic cancer) were also selected as non-hepatitis liver controls, and were retrospectively examined. The study was approved by the Jikei University Ethics Community Institutional Review Board.

Pathologic specimens. Tumor specimens were obtained by tumor biopsy with a $21-\mathrm{G}$ fine-needle aspiration kit. Nontumorous liver tissue specimens were concurrently obtained using an 18 - to $20-\mathrm{G}$ needle. Formalin-fixed and paraffinembedded liver tumor and non-tumor tissue specimens were processed for conventional histological assessment with hematoxylin and eosin staining. The tumors were histologically graded as either well or moderately differentiated.

Immunohistochemical analysis. Formalin-fixed paraffinembedded specimens were used for immunohistochemical analysis after deparaffinization. A rabbit anti-human survivin polyclonal antibody (Diagnostic Biosystems, USA) was used at a dilution of 1:2000 as the primary antibody, and detected with Envision+Rabbit/HRP (Dako, Japan). The specimens were heated in a microwave oven in antigen retrieval solution (10 mmol/l citrate buffer, $\mathrm{pH} 6.4$ ) at $121^{\circ} \mathrm{C}$ for $15 \mathrm{~min}$ for the retrieval of antigens, then cooled to room temperature. 3,3-Diaminobenzidine and hematoxylin were used for color 

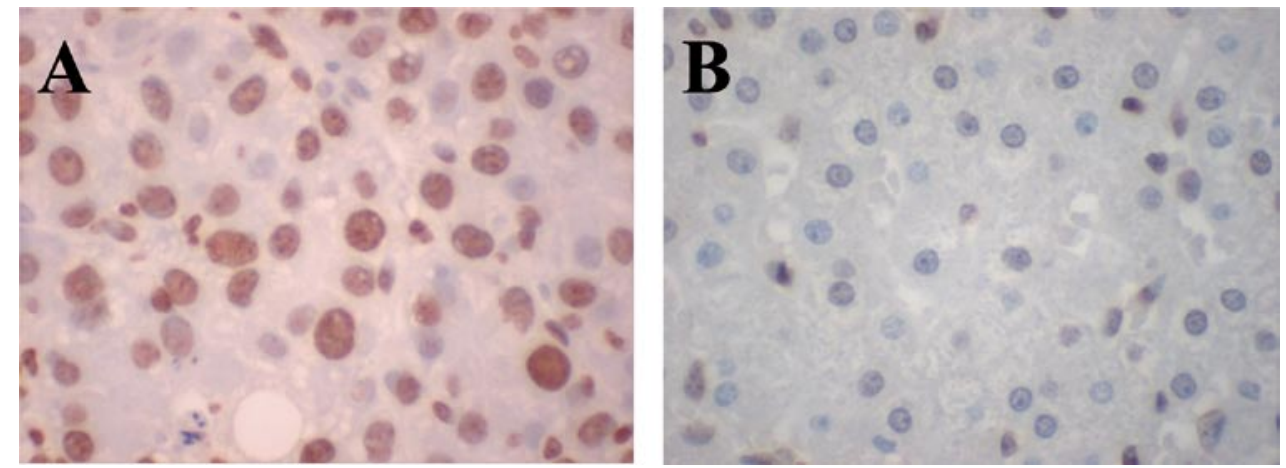

Figure 1. Immunohistochemical staining of survivin in hepatocellular carcinoma and peritumoral (hepatitis) biopsy tissues. (A) Tumor biopsy (x400); (B) Peritumoral biopsy (x400).

Table I. Characteristics of the patients undergoing tumor biopsy $(\mathrm{n}=22)$.

\begin{tabular}{lc}
\hline Features & Median value \\
\hline Age & $68(56-81)$ \\
Gender (male/female) & $15 / 7$ \\
Plt $\left(\times 10^{3} / \mu 1\right)$ & $10.0(5.1-24.5)$ \\
AST $(\mathrm{IU} / \mathrm{l})$ & $68(21-147)$ \\
ALT $(\mathrm{IU} / \mathrm{l})$ & $61.5(6-214)$ \\
T-Bil (mg/dl) & $0.8(0.4-2.3)$ \\
$\gamma-\mathrm{GTP}(\mathrm{IU} / \mathrm{l})$ & $48(18-665)$ \\
AFP $(\mathrm{ng} / \mathrm{ml})$ & $21.5(3-444)$ \\
HBs Ag/HCV Ab/others & $3 / 18 / 1$ \\
Tumor size (mm) & $14.5(8-23)$ \\
Cirrhosis (positive/negative) & $5 / 17$ \\
Differentiation (well/moderate) & $15 / 7$ \\
\hline
\end{tabular}

Data are expressed as the median (range) unless otherwise indicated. Hbs Ag, anti-hepatitis B surface antigen; HCV Ab, anti-hepatitis C antibody. Well, well-differentiated HCC; moderate, moderately differentiated HCC. Normal ranges: Plt (platelet count), 15-35x103/ $\mu 1$ AST (aspartate aminotransferase), 10-33 IU/l; ALT (alanine aminotransferase), 6-35 IU/1; T-Bil (total bilirubin), 0.2-1.2 mg/dl; $\gamma$-GTP ( $\gamma$-glutamyl transferase), 10-50 IU/l; AFP ( $\alpha$-fetoprotein), $>20 \mathrm{ng} / \mathrm{ml}$.

development and counterstaining, respectively. Cells with brown-colored nuclei were scored as positive. The mean percentage of survivin-positive HCC cells was determined in three areas at x100 magnification using the nuclear labeling index (labeled nuclei/500 nuclei). The same method was applied to hepatocytes in non-tumorous biopsy specimens.

Statistical analysis. Correlations between the degree of survivin-positive cells within biopsy tissues and clinicopathological variables were analyzed using Pearson's correlation coefficient. A multiple regression analysis, in which the explanatory variables were age, gender, Plt (platelet count), ALT (alanine aminotransferase), AFP ( $\alpha$-fetoprotein) and size of tumor, was performed to clarify the factors associated with survivin-positive cancer cell levels. Probabilities $<0.05$ were

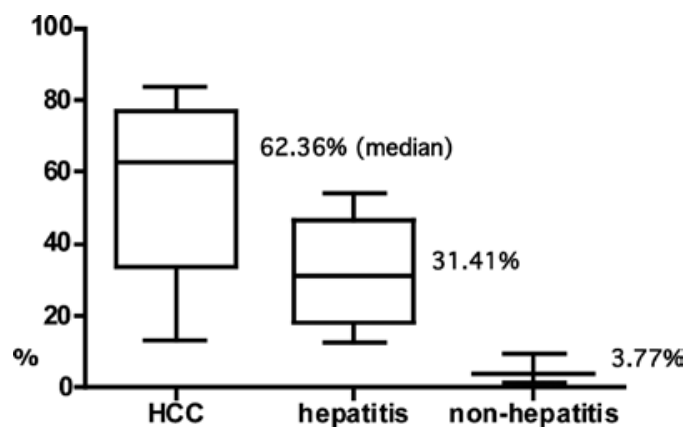

Figure 2. Survivin expression rates in hepatocellular carcinoma (HCC), hepatitis and non-hepatitis tissues.

considered to be statistically significant. The correlation analyses were performed using GraphPad Prism 4.00 (GraphPad Software, San Diego, USA), and multiple regression analysis was performed using the STATA 10.0 software program (STATA Corp., College Station, TX, USA).

\section{Results}

Survivin expression rates in early HCC and non-tumorous liver tissues. In HCC tissues, the rate of survivin expression was determined by counting survivin-positive cancer cells (Fig. 1A). The average survivin expression rate was $62.36 \%$ (median). In the peritumoral hepatitis tissues, the rate of survivin expression was determined in all hepatocytes (Fig. 1B). The average survivin expression rate was $31.41 \%$ (median). The survivin expression rate was also determined in all hepatocytes in the non-hepatitis tissues. The average survivin expression rate was $3.77 \%$ (median). Fig. 2 shows that survivin expression was higher in the tumor and peritumoral specimens than in the non-hepatitis tissues.

A correlation was also observed between the survivin expression rate in the peritumoral cells and the expression rate in $\mathrm{HCC}$ specimens $(\mathrm{r}=0.81, \mathrm{P}<0.001$, Fig. 3$)$.

Relationship between survivin expression rates and clinical parameters. Fig. 4 shows that the serum level of ALT was significantly correlated with the survivin expression rate in both the $\mathrm{HCC}$ specimens $(\mathrm{r}=-0.60, \mathrm{P}<0.01)$ and the non-tumorous liver tissues $(\mathrm{r}=-0.43, \mathrm{P}<0.05)$. No significant 


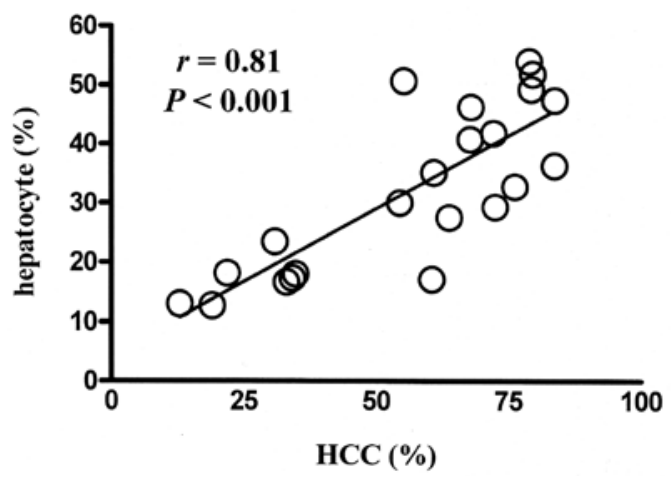

Figure 3. Correlation between the survivin expression rate in hepatocellular carcinoma (HCC) and peritumoral cells.

correlation was observed for other clinical parameters, such as the serum AFP level.

After adjustment for other covariates using multiple regression analysis, only the ALT level was thought to be a significant predictor of survivin expression $(\mathrm{P}=0.02$, Table II).

\section{Discussion}

Survivin, a member of the recently described IAP family, is characterized by a unique structure with a single BIR and no zinc-binding domain (14). It is undetectable in terminally differentiated adult tissues, but becomes notably expressed in the most common human cancers, including esophageal, stomach, colorectal, breast and pancreatic carcinoma (15-19). Survivin has also been implicated in the control of cell cycle kinetics and the inhibition of apoptosis (20-22). These findings suggest that survivin is an attractive novel target for anticancer interventions. Several preclinical studies have demonstrated that the down-regulation of survivin expression/function by means of an antisense oligonucleotide, dominant negative mutants, ribozymes, small interfering RNAs and cyclin-dependent kinase inhibitors increases the rate of apoptosis, reduces tumor growth potential and sensitizes tumor cells to various chemotherapeutic drugs and $\gamma$-irradiation in in vitro and in vivo models of various types of human tumors (12). YM155 was the first agent designed to inhibit survivin (23). Several early phase clinical studies demonstrated this novel anticancer agent to be well-tolerated and capable of shrinking recurrent
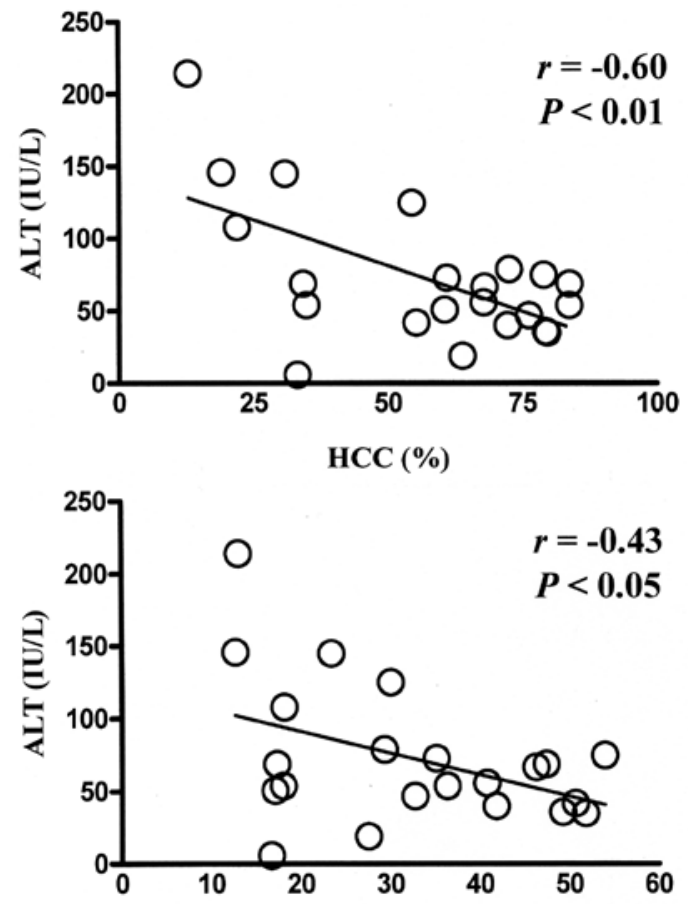

Figure 4. Correlation between serum levels of alanine aminotransferase (ALT) and survivin expression rates in hepatocellular carcinoma (HCC) and peritumoral cells.

tumors in some patients with non-Hodgkin lymphoma and hormone-refractory prostate cancer after conventional chemotherapy. In addition, interim reports indicate that it has few side effects. It is possible that survivin inhibition in early HCC could be potentially useful as an effective interventional radiological treatment modality (13). Moreover, the current study showed the expression of survivin in HCC to be correlated with peritumoral hepatocytes and serum ALT levels. This suggests that patients with HCC could benefit from survivin inhibitive therapy, even in cases where liver function has been stabilized.

The expression of survivin has been detected in a variety of preneoplastic and/or benign lesions, including polyps of the colon, breast adenomas, Bowen's disease and hypertrophic actinic keratosis (24). This suggests that the expression of survivin may occur during early malignant transformation

Table II. Results of a multiple regression analysis associated with the survivin-positive rates of hepatocellular carcinoma tissues.

\begin{tabular}{lcccc}
\hline Clinicopathological variables & Coefficient & Standard error & 95\% Confidence interval of coefficient & P-value \\
\hline Age & 0.004790 & 0.006960 & $-0.0100-0.0196$ & 0.501 \\
Gender & 0.076200 & 0.143000 & $-0.228-0.380$ & 0.601 \\
Plt & -0.004020 & 0.011900 & $-0.0294-0.0214$ & 0.740 \\
ALT & -0.002710 & 0.001040 & $-0.004930--0.000484$ & $\mathbf{0 . 0 2 0}$ \\
AFP & -0.000554 & 0.000438 & $-0.00149-0.00380$ & 0.225 \\
Tumor size & 0.000677 & 0.012500 & $-0.0260-0.0274$ & 0.958
\end{tabular}

Plt, platelet count; ALT, alanine aminotransferase; AFP, $\alpha$-fetoprotein. 
or following a disturbance in the balance between cell proliferation and cell death (12). A previous study showed that the HBV X and HCV core proteins activate NF- $\mathrm{KB}$ and/or STAT-3, which regulate gene expression for cell survival factors such as anti-apoptotic proteins, including survivin $(25,26)$. In the current study, the average survivin expression in peritumoral liver tissues was $31.41 \%$ (median). Survivin expression may also occur in the presence of the hepatitis virus (13). In this study, survivin expression was correlated with the serum levels of ALT. These findings indicate that, in viral hepatitis, survivin expression induces early malignant transformation following a disturbance in the balance between cell proliferation and cell death, even if liver function has been stabilized.

In 1955, elevations in serum AST levels were reported in viral hepatitis and other hepatic diseases, Subsequently, concomitant ALT elevations were found in similar disorders. AST and ALT are abundant hepatic enzymes that catalyze the transfer of amino groups to form the hepatic metabolites pyruvate and oxaloacetate, respectively. ALT is found in the cytosol in liver cells, whereas the two AST isoenzymes are located in the cytosol and mitochondria, respectively. Both ALT and AST are released from damaged hepatocytes into the blood after hepatocellular injury or death. AST is also abundantly expressed in several non-hepatic tissues, including the heart, skeletal muscle and blood. ALT is found at low concentrations in tissues other than the liver, and is therefore frequently considered specific for hepatocellular injury (27). In this study, a negative correlation between the rate of survivin expression and the serum level of ALT was observed in $\mathrm{HCC}$ and hepatocytes in patients with hepatitis. This suggests that the expression of survivin occurs with the inhibition of apoptosis, or following a disturbance in the balance between cell proliferation and cell death. ALT was not significantly released into the blood by damaged HCCs or hepatocytes, suggesting that the expression of survivin inhibits cellular injury or death caused by HCC and hepatitis.

Scattered HCCs are commonly treated by the embolization of the supplying branch of the hepatic artery, or by direct hepatic artery injection of chemotherapeutic agents followed by local embolization. The main advantage of TACE is that it allows the local administration of anticancer drugs to a specific cancerous area. In this study, a correlation was observed between the rate of survivin expression in peritumoral cells and the rate of expression in HCC specimens $(r=0.81, \mathrm{P}<0.001)$. The average rate of survivin expression was $31.41 \%$ (median) in the peritumoral area. If side effects are taken into consideration with survivin inhibitive therapy, then interventional radiological treatment of a selective artery with a survivin inhibitor could be an effective therapy for scattered HCCs.

In conclusion, the expression of survivin was analyzed during the early stages of HCC additionally surrounded by hepatitis, and the correlation of this expression with clinicopathological factors was measured. The serum level of ALT was correlated with the rate of survivin expression in the HCC and peritumoral hepatitis specimens. Multiple regression analysis showed that only serum ALT levels were associated with survivin expression in HCC. These findings suggest that various clinicopathological factors may in future serve as useful indicators for the selection of patients responsive to the inhibition of survivin.

\section{References}

1. Parkin DM: International variation. Oncogene 23: 6329-6340, 2004.

2. Hiraoka A, Kumagi T, Hirooka M, Uehara T, Kurose K, Iuchi H, Hiasa Y, Matsuura B, Michitaka K, Kumano S, Tanaka H, Yamashita Y, Horiike N, Mochizuki T and Onji M: Prognosis following transcatheter arterial embolization for 121 patients with unresectable hepatocellular carcinoma with or without a history of treatment. World J Gastroenterol 12: 2075-2079, 2006.

3. Ye CP, Qiu CZ, Huang ZX, Su QC, Zhuang W, Wu RL and Li XF: Relationship between survivin expression and recurrence, and prognosis in hepatocellular carcinoma. World J Gastroenterol 13: 6264-6268, 2007.

4. Altieri DC and Marchisio PC: Survivin apoptosis: an interloper between cell death and cell proliferation in cancer. Lab Invest 79: 1327-1333, 1999.

5. Giodini A, Kallio MJ, Wall NR, Gorbsky GJ, Tognin S, Marchisio PC, Symons M and Altieri DC: Regulation of microtubule stability and mitotic progression by survivin. Cancer Res 62: 2462-2467, 2002.

6. Wakana Y, Kasuya K, Katayanagi S, Tsuchida A, Aoki T, Koyanagi Y, Ishii $\mathrm{H}$ and Ebihara Y: Effect of survivin on cell proliferation and apoptosis in gastric cancer. Oncol Rep 9: 1213-1218, 2002.

7. Lu M, Kwan T, Yu C, Chen F, Freedman B, Schafer JM, Lee EJ, Jameson JL, Jordan VC and Cryns VL: Peroxisome proliferatoractivated receptor gamma agonists promote TRAIL-induced apoptosis by reducing survivin levels via cyclin D3 repression and cell cycle arrest. J Biol Chem 280: 6742-6751, 2005.

8. Tarnawski A, Pai R, Chiou SK, Chai J and Chu EC: Rebamipide inhibits gastric cancer growth by targeting survivin and Aurora-B. Biochem Biophys Res Commun 334: 207-212, 2005.

9. Kania J, Konturek SJ, Marlicz K, Hahn EG and Konturek PC: Expression of survivin and caspase-3 in gastric cancer. Dig Dis Sci 48: 266-271, 2003.

10. Zhu XD, Lin GJ, Qian LP and Chen ZQ: Expression of survivin in human gastric carcinoma and gastric carcinoma model of rats. World J Gastroenterol 9: 1435-1438, 2003.

11. Ikeguchi M, Liu J and Kaibara N: Expression of survivin mRNA and protein in gastric cancer cell line (MKN-45) during cisplatin treatment. Apoptosis 7: 23-29, 2002.

12. Zaffaroni N, Pennati M and Daidone MG: Survivin as a target for new anticancer interventions. J Cell Mol Med 9: 360-372, 2005.

13. Mamori S, Asakura T, Ohkawa K and Tajiri H: Survivin expression in early hepatocellular carcinoma and post-treatment with anti-cancer drug under hypoxic culture condition. World J Gastroenterol 13: 5306-5311, 2007.

14. Span PN, Tjan-Heijnen VC, Heuvel JJ, De Kok JB, Foekens JA and Sweep FC: Do the survivin (BIRC5) splice variants modulate or add to the prognostic value of total survivin in breast cancer? Clin Chem 52: 1693-1700, 2006.

15. Ikeguchi M and Kaibara N: Survivin messenger RNA expression is a good prognostic biomarker for oesophageal carcinoma. Br J Cancer 87: 883-887, 2002.

16. Miyachi K, Sasaki K, Onodera S, Taguchi T, Nagamachi M, Kaneko $\mathrm{H}$ and Sunagawa M: Correlation between survivin mRNA expression and lymph node metastasis in gastric cancer. Gastric Cancer 6: 217-224, 2003.

17. Sarela AI, Scott N, Ramsdale J, Markham AF and Guillou PJ: Immunohistochemical detection of the anti-apoptosis protein, survivin, predicts survival after curative resection of stage II colorectal carcinomas. Ann Surg Oncol 8: 305-310, 2001.

18. Ryan B, O'Donovan N, Browne B, O'Shea C, Crown J, Hill AD, McDermott E, O'Higgins N and Duffy MJ: Expression of survivin and its splice variants survivin-2B and survivin-DeltaEx3 in breast cancer. Br J Cancer 92: 120-124, 2005.

19. Sarela AI, Verbeke CS, Ramsdale J, Davies CL, Markham AF and Guillou PJ: Expression of survivin, a novel inhibitor of apoptosis and cell cycle regulatory protein, in pancreatic adenocarcinoma. Br J Cancer 86: 886-892, 2002.

20. O'Connor DS, Grossman D, Plescia J, Li F, Zhang H, Villa A, Tognin S, Marchisio PC and Altieri DC: Regulation of apoptosis at cell division by p34cdc2 phosphorylation of survivin. Proc Natl Acad Sci USA 97: 13103-13107, 2000.

21. Fukuda S, Mantel CR and Pelus LM: Survivin regulates hematopoietic progenitor cell proliferation through p21WAF1/ Cip1-dependent and -independent pathways. Blood 103: 120-127, 2004. 
22. Rosa J, Canovas P, Islam A, Altieri DC and Doxsey SJ: Survivin modulates microtubule dynamics and nucleation throughout the cell cycle. Mol Biol Cell 17: 1483-1493, 2006.

23. Nakahara T, Takeuchi M, Kinoyama I, Minematsu T, Shirasuna K, Matsuhisa A, Kita A, Tominaga F, Yamanaka K, Kudoh M and Sasamata M: YM155, a novel small-molecule survivin suppressant, induces regression of established human hormone-refractory prostate tumor xenografts. Cancer Res 67: 8014-8021, 2007.
24. Altieri DC: Survivin, versatile modulation of cell division and apoptosis in cancer. Oncogene 22: 8581-8589, 2003.

25. Waris $\mathrm{G}$ and Siddiqui A: Regulatory mechanism of viral hepatitis B and C. J Biosci 28: 311-321, 2003.

26. Kountouras J, Zavos C and Chatzopoulos D: Apoptosis in hepatitis C. J Viral Hepat 10: 335-342, 2003.

27. Green RM and Flamm S: AGA technical review on the evaluation of liver chemistry tests. Gastroenterology 123: 1367-1384, 2002. 